\title{
MONITOREO DE DAÑOS Y ANÁLISIS NUMÉRICO EN UN EDIFICIO HISTÓRICO AFECTADO POR UNA FALLA SUPERFICIAL ACTIVA EN EL VALLE DE AGUASCALIENTES
}

\author{
Martín Hernández Marín ${ }^{(1)}$, Hugo David Montalvo Delgado ${ }^{(2)}$, José Ángel Ortiz Lozano $^{(1)}$ \\ María de Jesús Láriz Medina ${ }^{(2)}$, Jesús Pacheco Martínez ${ }^{(1)}$ y Mario Eduardo Zermeño de León ${ }^{(1)}$
}

\begin{abstract}
RESUMEN
El Museo de la Insurgencia, declarado edificio histórico y patrimonial y localizado en Pabellón de Hidalgo, estado de Aguascalientes, México central, está siendo severamente dañado por una falla superficial activa. Para este trabajo se implementó una metodología sencilla para monitorear y cuantificar los desplazamientos derivados de la falla a partir de los cuales se realizó un análisis lineal de esfuerzos mediante simulaciones numéricas para evaluar daños estructurales, identificar puntos críticos y realizar una predicción aproximada de situación estructural crítica en un futuro próximo. La metodología incluye una revisión de la última adecuación estructural del edificio diseñada para que éste conviviera con la falla que lo afecta. Utilizando fisurómetros colocados estratégicamente en los elementos constructivos del museo a lo largo de la línea de falla se logró la evaluación cuantitativa de los desplazamientos inducidos por la falla. El monitoreo se realizó por un lapso de 202 días obteniéndose desplazamientos verticales y horizontales máximos de $6 \mathrm{~mm}$ y $10 \mathrm{~mm}$, respectivamente. Los resultados de las simulaciones numéricas permitieron determinar que, bajo las condiciones dinámicas actuales del efecto de la falla, el edificio está siendo críticamente dañado en las bases de las columnas más cercanas a la falla, de tal modo que en un periodo de mediano plazo los esfuerzos inducidos por la falla sobrepasarán la resistencia del edificio en algunos puntos.
\end{abstract}

Palabras clave: Falla geológica; Museo de la Insurgencia; valle de Aguascalientes; análisis numérico

\section{MONITORING DAMAGES AND NUMERICAL ANALYSIS IN A HISTORIC BUILDING AFFECTED BY AN ACTIVE SURFACE FAULT IN THE VALLEY OF AGUASCALIENTES}

\begin{abstract}
The Museum of the Insurgence, inherited historic building located in the community of Pabellon de Hidalgo, Aguascalientes state, central Mexico, is been severely damaged by an active surface fault. In this work, a simple methodology was implemented to quantify and monitor fault-induced

Artículo recibido el 30 de septiembre de 2014 y aprobado para su publicación el 7 de julio de 2016. Se aceptarán comentarios y/o discusiones hasta cinco meses después de su publicación.

(1) Centro de Ciencias del Diseño y de la Construcción, Universidad Autónoma de Aguascalientes, Avenida Universidad No. 940, Ciudad Universitaria, Aguascalientes, Aguascalientes, México, C.P. 20131. mhernandez@correo.uaa.mx; aortiz@correo.uaa.mx; jesus.pacheco@edu.uaa.mx; mezerme@correo.uaa.mx

(2) Maestría en Ingeniería Civil, Universidad Autónoma de Aguascalientes, Avenida Universidad No. 940, Ciudad Universitaria, Aguascalientes, Aguascalientes, México, C.P. 20131. montalvo 1964gt@hotmail.com; maritza.lariz@gmail.com
\end{abstract}


displacements that are used to perform a stress lineal analysis through numerical modeling to assess structural damages, identify critical points, and perform a future approximated prediction of the structural critical situation in a short term. The methodology includes a revision of the last structural restoration of the building, which was designed for the coexistence between the building and the fault that affects it. By placing crack gauges in strategic structural elements of the building along the fault trace, the quantifying evaluation of the fault-induced displacements was achieved. Displacements were measured in a lapse of 202 days reaching values equal to $6 \mathrm{~mm}$ and $10 \mathrm{~mm}$ vertically and horizontally, respectively. Based on the results of the modeling analyses, which consider the actual dynamic conditions of the fault effect, the building is being critically damaged on the base of the masonry columns closer to the fault, where in a mid-term period, fault-induced stresses may exceed the masonry strength.

Keywords: Geologic fault; Museum of the Insurgence; valley of Aguascalientes; numerical analysis

\section{INTRODUCCIÓN}

Varias construcciones dentro del valle de Aguascalientes presentan diferentes grados de afectación estructural como consecuencia directa o indirecta de la incidencia de fallas y fracturas superficiales, las cuales a su vez se presentan en conjunto con el fenómeno de subsidencia. Una de estas construcciones es el Museo de la Insurgencia, anteriormente llamada Hacienda de San Blas, localizada en la comunidad de Pabellón de Hidalgo, cercana a la ciudad de Aguascalientes, México. Durante la guerra de independencia, este edificio jugó un importante rol histórico dado que fue en este lugar en el que el independentista Miguel Hidalgo y Costilla fue relegado de su posición dentro del ejército por los generales de la rebelión. Por este hecho y otros de alto valor histórico, este edificio en la actualidad cuenta con la categoría de patrimonio histórico mundial. La Figura 1 muestra la localización del Museo de la Insurgencia.

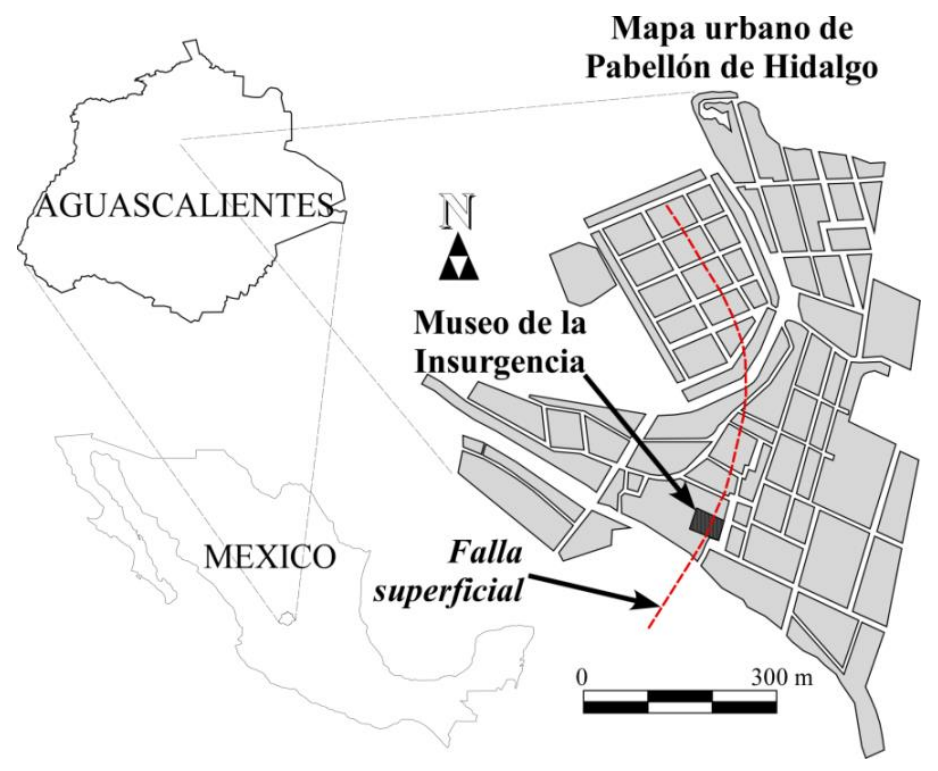

Figura 1. Localización del Museo de la Insurgencia.

Este edificio es peculiar porque su estructura ha sido recientemente adaptada para soportar los efectos de una falla geológica superficial que actualmente le está causando daño. La idea original de esta restauración estructural fue la de permitir al edificio coexistir con la falla con el fin de preservarlo. Sin 
embargo, la falla superficial que le afecta sigue causando severo daño mediante continuos desplazamientos, los cuales inducen la aparición de grietas en las columnas y muros adyacentes a la falla, así como una continua separación de unos arcos de mampostería. En este manuscrito, el término falla será utilizado cuando los desplazamientos observados sean tanto verticales como horizontales, mientras que el término fractura denotará únicamente desplazamientos horizontales perpendiculares a su traza.

Un estudio previo sobre las afectaciones en construcciones por fallas y fracturas en el Valle de Aguascalientes, principalmente viviendas, demostró alta variabilidad en las patologías de daños (OrtizLozano et al., 2010). Sin embargo, en ese estudio el rol real de estas fallas y fracturas no fue conclusivo porque algunos de estos daños fueron atribuidos en parte a materiales defectuosos o de mala calidad utilizados en la construcción, así como a inadecuados métodos constructivos. La investigación de OrtizLozano et al. (2010) junto con otros casos más recientes son incluidos y discutidos en más detalle en el trabajo presentado por Pacheco-Martínez et al. (2013). Para el 2010, se reportaron un total de 209 fallas y fracturas superficiales dentro del valle de Aguascalientes, completando una longitud total acumulada de 316,374 metros lineales y causando severos daños en más de 2,000 edificios (Secretaría de Obras Públicas, 2010). Dentro del valle de Aguascalientes, los daños en construcciones asociados con fallas y fracturas geológicas contienen inherente interés socioeconómico, debido principalmente a los daños asociados por reparaciones o pérdida total, o por el evidente riesgo por colapso repentino de muros o losas que un edificio dañado representa a sus residentes, quienes en algunos casos tienen que abandonar su vivienda.

En un estudio reciente sobre la dinámica de la falla que afecta la comunidad de Pabellón de Hidalgo, se obtuvieron datos de desplazamiento mediante fisurómetros, los cuales fueron estratégicamente colocados en varios elementos constructivos afectados a lo largo de la falla, principalmente muros y guarniciones de calles (Láriz-Medina, 2013). El análisis de los datos resultantes mostró alta heterogeneidad en los desplazamientos de la falla, con magnitudes mayores hacia el sur de la comunidad, en donde se localiza el Museo de la Insurgencia. De un censo elaborado en el 2013 para la comunidad de Pabellón de Hidalgo, se estimó una población de 4,002 habitantes, los cuales habitaban 896 hogares (Nuestro México.com, 2013). La única falla que afecta la población de Pabellón de Hidalgo tiene una longitud aproximada de 913 metros, cruzando casi en su totalidad la zona urbana y causando daños en más de 30 construcciones incluyendo casas habitación, iglesias, escuelas y el Museo (Láriz-Medina, 2013). Como puede observarse en la figura 1 , esta falla en particular tiene una orientación de $65^{\circ} \mathrm{SO}-\mathrm{NE}$ en su parte sur, y de $24^{\circ} \mathrm{SE}-\mathrm{NO}$ en el norte, siguiendo aproximadamente la configuración de las líneas topográficas. La fecha del origen de esta falla no está clara, pero considerando que la mayoría de las fracturas y fallas dentro del valle aparecieron en los 1980's (Aranda-Gómez, 1989; Aranda-Gómez y Aranda-Gómez, 1985), se puede asumir ese inicio aproximado para esta falla en particular.

En cuanto a la modelación numérica de edificios históricos, que incluye la técnica de elementos finitos, Orduña et al. (2007) mencionan que aun con las técnicas y teorías de análisis actuales, esta técnica sigue siendo un desafío por diversos factores, sobre todo debido a las características de la mampostería al tratarse de un material compuesto con alta resistencia a la compresión pero muy baja a la tensión. En ese contexto, Peña-Mondragón (2010) menciona que además de esos factores propios de la estructura física, se debe tener especial cuidado con otros factores numéricos, como lo son la descripción geométrica, el diseño estructural, la buena identificación de los materiales utilizados en la construcción, así como de las propiedades mecánicas y calidad de dichos materiales. Por otro lado, de acuerdo con Creazza et al. (2002) para el análisis de este tipo de edificios de mampostería como el Museo de la Insurgencia, existen dos aproximaciones generales: el micromodelado que está definido por la utilización de elementos continuos de las piezas y las juntas, y macromodelado que representa a la mampostería como un solo medio homogéneo (López et al., 1998). En cuanto al grado de complejidad de un modelo numérico que incluye aproximaciones lineales y no lineales, Lourenço (2001) recomienda realizar modelos simples lineales para análisis 
preliminares y tener un conocimiento del comportamiento global de la estructura. Aunque como lo mencionan Meli y Sánchez (1996) y Meli (1998) las consideraciones sobre el análisis estructural de estructuras de mampostería deben realizarse con una metodología muy diferente a las estructuras actuales basadas en elementos de concreto y acero.

La meta general de este trabajo es discutir las afectaciones estructurales que una falla superficial activa presenta sobre un edificio, en este caso el Museo de la Insurgencia en el estado de Aguascalientes, México. Se analizan los resultados de las simulaciones numéricas, las cuales consideran los desplazamientos registrados por efecto de una falla geológica superficial en el Museo. Las simulaciones se plantearon inicialmente para detectar las zonas con el daño estructural más crítico, y luego usando un análisis estadístico de tendencias de desplazamiento implementado en el modelo numérico, se realizó una comparación en los arcos de mampostería sobre de los esfuerzos simulados y los permitidos dentro del estado límite de falla, ya que como se observó y como se discute más adelante, las bases de los arcos representan los elementos afectados más críticos en el Museo. Los resultados de la evaluación de un escenario futuro en esta investigación podrían ser utilizados para proponer periodos de futura renovación estructural al edificio si los efectos de la falla convirtieran la estructura inestable. Este estudio representa quizás uno de los primeros en su tipo sobre edificios que inicialmente son adaptados para coexistir con una falla geológica, pero con daños posteriores debido a la continua dinámica de la falla.

\section{ESTADO ESTRUCTURAL ACTUAL E HISTÓRICO}

Debido a su valor histórico y cultural, el "Camino Real Tierra Adentro", que abarca varios estados de México y Texas en Estados Unidos, en una ruta de longitud cercana a los $2600 \mathrm{~km}$, fue denominado por la UNESCO como patrimonio histórico y cultural de la humanidad. Este camino incluye una serie de monumentos y sitios históricos entre los que se encuentra el Museo de la Insurgencia. Por esta razón principal, se han realizado una serie de acciones y adecuaciones estructurales para preservarlo, a sabiendas de que una falla superficial activa le está causando severo daño.

La figura 2 muestra algunas características arquitectónicas del Museo de la Insurgencia en donde además se puede apreciar la línea de la falla superficial que afecta al edificio, mientras que la figura 3 muestra la principal fachada del Museo. La línea de la falla cruza el edificio completamente de norte a sur, separándolo en dos bloques este y oeste, como se aprecia en la figura 2. Debido a esto, las adecuaciones constructivas realizadas al edificio con fines de preservación le han permitido eventualmente coexistir con la falla. Sin embargo, debido a la dinámica natural de la falla, el edificio podría estar en peligro de colapso en un futuro próximo. Las adaptaciones y refuerzos que se realizaron en el edificio pueden resumirse en dos. En la primera y estructuralmente más importante, se estableció una junta fría de unos 10 milímetros sobre lo que hasta ese entonces se distinguió como la traza de la falla. En esta primera adaptación los muros, techos y arcos tanto norte como sur fueron separados en dos para permitir su libre desplazamiento y mitigar o evitar la concentración de esfuerzo en dichos elementos. Para evitar el colapso de los arcos norte y sur, se colocó una viga IR de acero de $254 \mathrm{~mm}$ y $44.8 \mathrm{~kg} / \mathrm{m}$ unida por una solera, de acuerdo a las observaciones (figura 8). Este elemento estructural atraviesa casi en su totalidad el trazado de los arcos, por lo que a partir de entonces este elemento metálico está soportando todos los esfuerzos inducidos. Después de establecida esta primer junta, el edificio quedó prácticamente divido en dos bloques por unos $10 \mathrm{~mm}$, pero con el fin de evitar infiltraciones por el techo durante las lluvias, se colocó una lámina de metal sobre el espacio de la junta. La figura 4 muestra algunos aspectos de los arcos norte y su adaptación estructural, incluyendo la lámina de metal en el techo para evitar infiltraciones. 

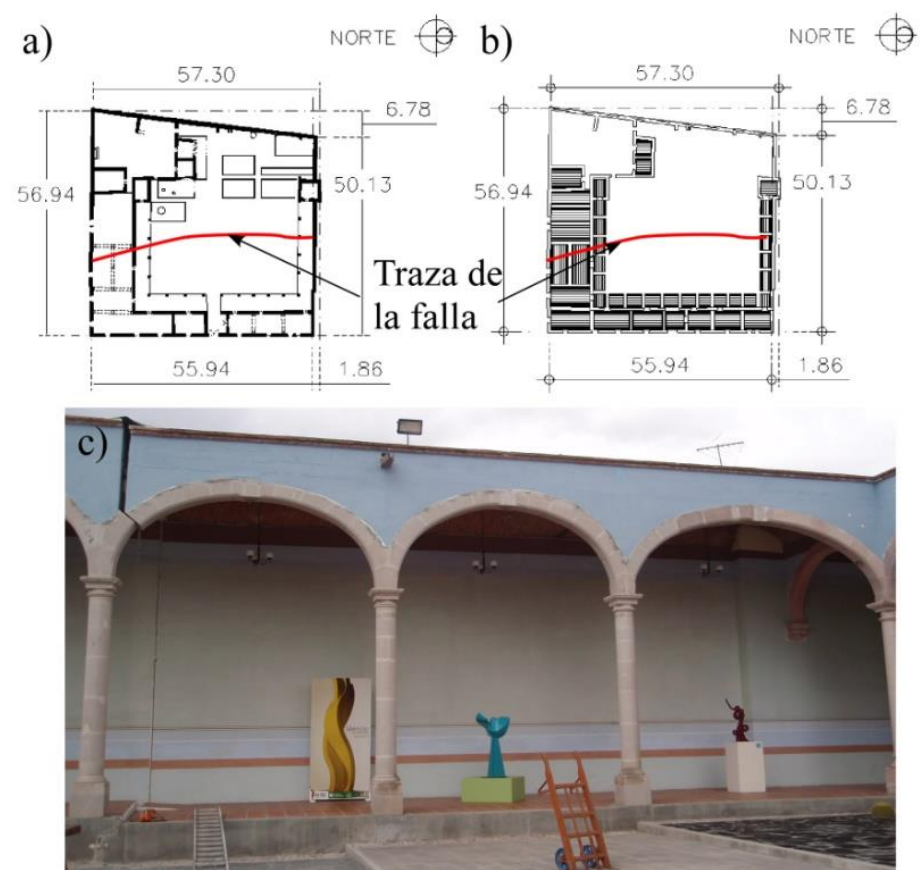

Figura 2. Planos arquitectónicos en planta y fotografía de los arcos internos. El plano a) muestra la distribución arquitectónica, el plano b) muestra un plano del techo. La foto c) muestra los arcos norte, desde el patio central.

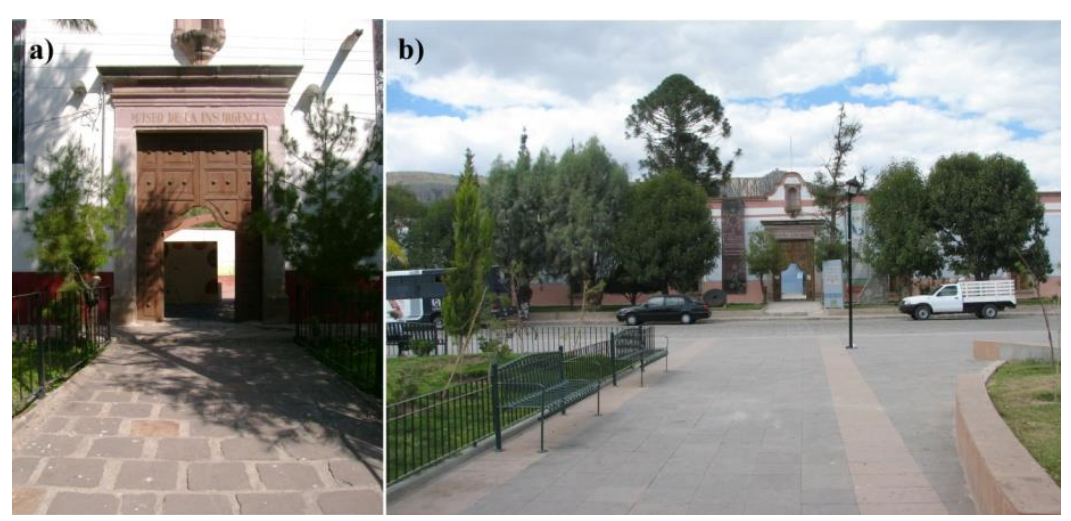

Figura 3. Imagen de la fachada del Museo de la Insurgencia 


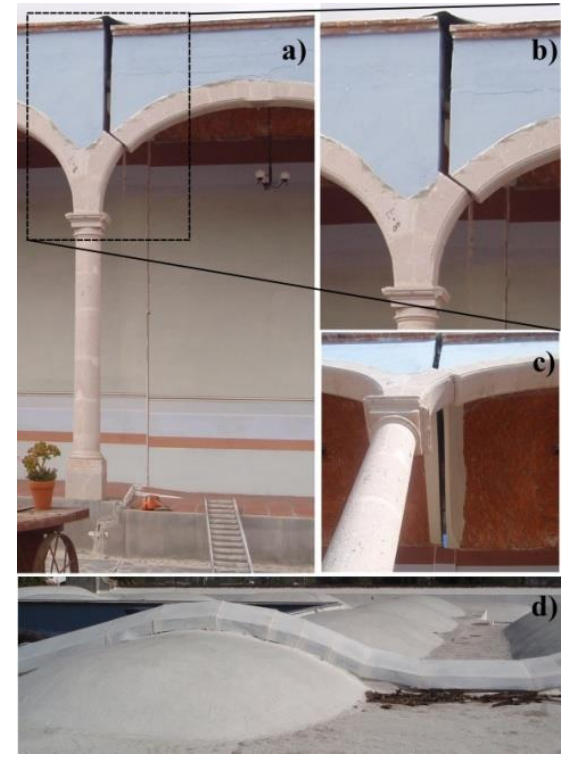

Figura 4. Detalle de la junta establecida los arcos norte y el laminado en el techo para evitar la entrada de agua pluvial. a), b) y c) muestran la junta en los elementos de los arcos norte, d) el techo del edificio.

La segunda adaptación consistió en establecer otra junta fría en el patio central también de unos 10 milímetros, por lo que después de esta junta, el edificio quedó completamente dividido en dos bloques. Es de recalcar que, en un principio, esta última junta coincidía con la traza de falla, pero que en la actualidad difiere en casi un metro de la junta en algunos puntos, como se observa en la figura 5. Este cambio podría deberse a que el efecto de las heterogeneidades en el suelo se acentúa conforme el tiempo transcurre, además de que los esfuerzos podrían estar incidiendo con diferente magnitud en varios puntos de la falla. Al igual que en el patio central, la junta de los pasillos fue rellenada con membrana de silicón también para evitar daños por infiltraciones.
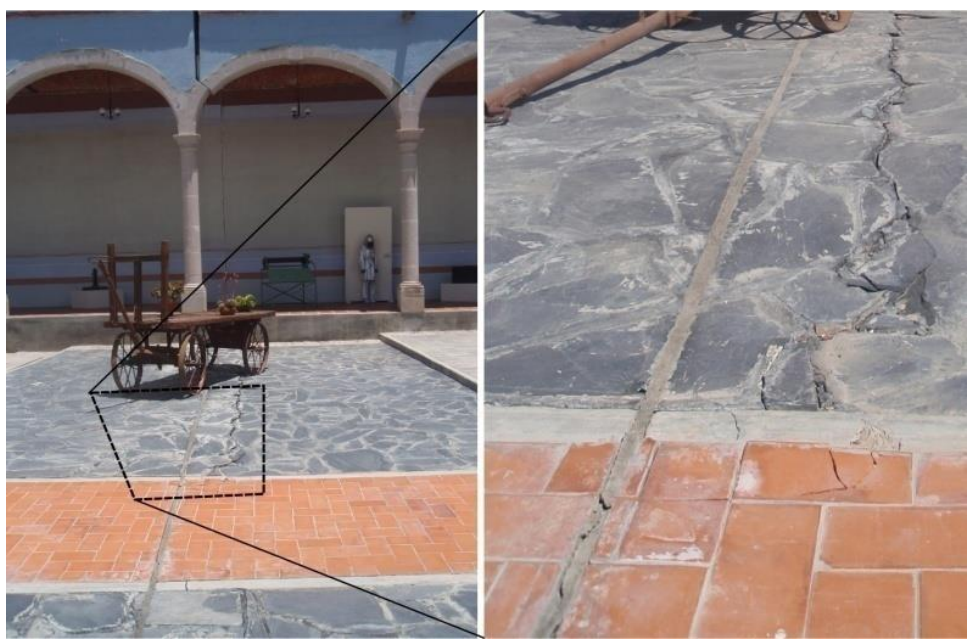

Figura 5. Efectos de la falla en el piso del patio central. En la fotografía ampliada se puede apreciar, a la derecha de la junta fría, la actual traza de la falla (ruptura de pisos). 


\section{METODOLOGÍA}

Las actividades diseñadas para lograr las metas de esta investigación se desarrollaron de la siguiente manera:

1. Determinación de la condición estructural del edificio. Aunque desafortunadamente la información importante sobre su diseño estructural, tanto del diseño original como de la adaptación estructural reciente no están disponibles, por lo que la información presentada en este trabajo está basada en observaciones y mediciones superficiales directas sobre el edificio.

2. Análisis de la dinámica de la falla utilizando datos de desplazamientos relativos registrados en los diferentes fisurómetros colocados en el edificio. Se colocaron 5 fisurómetros o testigos de manufactura propia en los diferentes elementos afectados a lo largo de la falla, como se muestra en la figura 6, sin embargo, para el análisis sólo se utilizaron los primeros cuatro dado que el quinto presentó fallas. Estos fisurómetros consisten en dos láminas simples de acrílico con espesor de $3 \mathrm{~mm}$. Cada una de estas láminas lleva adherida una película de acetato, una con un cuadrante con sistema de coordenadas cartesianas y la otra con una cruz emulando un puntero. El acetato con el cuadrante lleva grabada una cuadrícula graduada en milímetros, aunque enumerando solamente los centímetros. Cada uno de estos fisurómetros se colocaron de modo que cada lámina que los constituye, estuviera posicionada en cada bloque de separación, permitiendo que los desplazamientos derivados directamente por la falla fueran registrados. Dado que los fisurómetros fueron colocados cruzando la traza de la falla, sólo se capturaron las componentes de desplazamiento con direcciones vertical y horizontal perpendicular a la traza de la falla, es decir, no se capturaron los desplazamientos que pudieran ocurrir en la misma dirección de la traza de la falla, los cuales son comunes en fallas transcurrentes o mixtas.
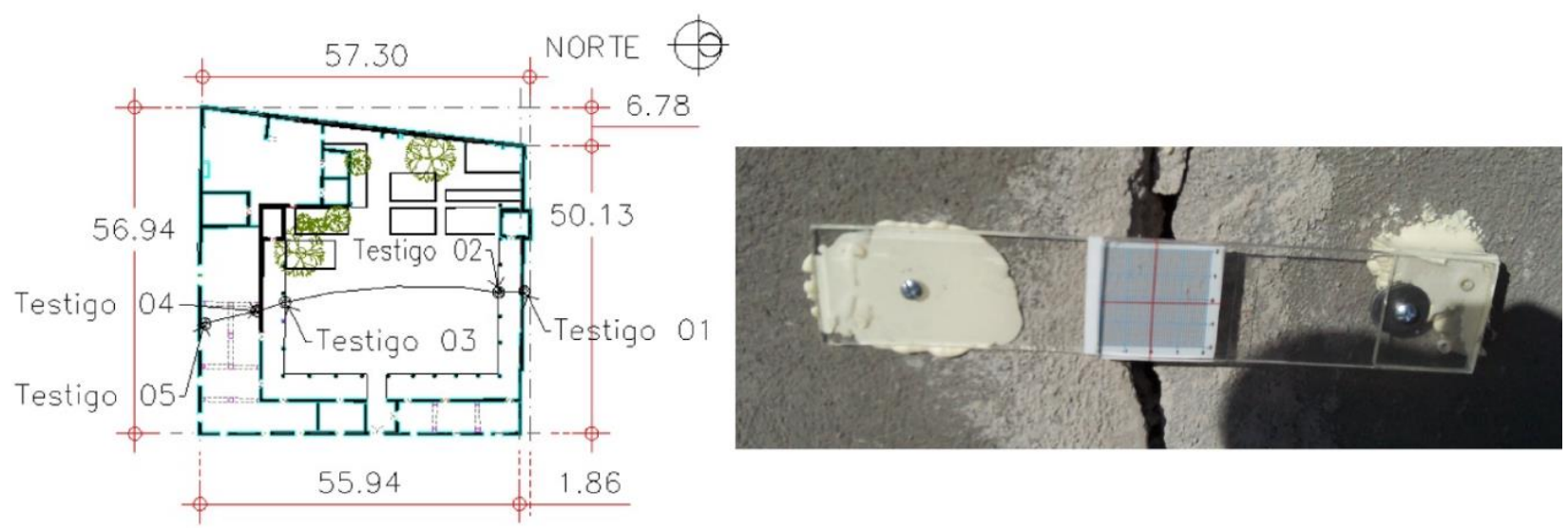

Figura 6. Diagrama en planta del edificio con la ubicación de los fisurómetros (testigos 01 al 05). En la imagen derecha se muestra el fisurómetro 02 colocado en la fractura de la mampostería.

3. Los resultados de las deformaciones se utilizaron como datos de entrada en las simulaciones numéricas. La idea de la investigación fue la de establecer, mediante simulaciones numéricas, la ubicación de las zonas críticas de máxima acumulación de esfuerzos derivados directamente de la incidencia de la falla. Las simulaciones fueron útiles además para evaluar el tiempo crítico aproximado en el que los esfuerzos máximos incidentes en el edificio sobrepasarán los permisibles. Este último análisis se basó en la tendencia de desplazamientos, bajo el criterio de que ésta se mantenga fija en el futuro. Se utilizaron modelos lineales elásticos, utilizando el software comercial SAP2000. Este software permite el análisis completo de desempeño estructural, además de que 
permite incluir datos de desplazamientos como datos de entrada y obtener los esfuerzos resultantes, cuyos valores más críticos, tanto actuales como futuros fueron comparados con los estados límites de falla permitidos en los reglamentos de construcción vigentes. Se llevó a cabo un análisis elástico lineal debido a que el objetivo primordial del estudio era medir el nivel de esfuerzos del edificio en función de los desplazamientos generados por la falla, pero de una forma simplificada y aproximada.

\section{Simulación y análisis de la situación actual}

Para analizar la respuesta estructural de los arcos y columnas en los modelos numéricos se consideraron las cargas vivas y cargas muertas que inciden en estos elementos por acciones gravitacionales estáticas. Para la estimación de las cargas muertas se utilizaron los pesos volumétricos de los materiales empleados en la edificación del inmueble, sin embargo, dado que el inmueble presenta muy baja frecuencia de incidencia de cargas vivas en su techo, se determinó utilizar un valor relativamente bajo de $0.29 \mathrm{kPa}$. Las cargas muertas se estimaron considerando un peso volumétrico de $1,500 \mathrm{~kg} / \mathrm{m}^{3}$ y un espesor promedio de $25 \mathrm{~cm}$ con un ancho tributario de $2 \mathrm{~m}$, resultando en una carga muerta de $7.36 \mathrm{kPa}$, la cual es soportada por la viga IR unida por la solera. La tabla 1 muestra los parámetros de los materiales utilizados en la construcción y aplicados a las simulaciones. Finalmente, se incluyeron los desplazamientos inducidos por la falla geológica, de los cuales se tomó el acumulado en un lapso de 202 días de monitoreo. En SAP2000 es posible determinar esfuerzos en elementos estructurales a partir de un desplazamiento. La sumatoria de cargas se puede observar de manera simple mediante la ecuación 1.

$\mathrm{C}=\mathrm{PP}+\mathrm{CM}+\mathrm{CV}+\mathrm{FG}$

donde PP es el peso propio de la estructura, CM la sumatoria de las cargas muertas, CV de las cargas vivas y FG representa las acciones mecánicas debidas a los desplazamientos verticales, inducidas por la falla geológica y las obtenidas mediante las simulaciones numéricas. Este último parámetro es de magnitud variable de acuerdo con los desplazamientos diferenciales registrados en el edificio.

Tabla 1. Propiedades mecánicas de los materiales empleados en la construcción y rehabilitación del Museo de la Insurgencia

\begin{tabular}{ccccccc}
\hline Elemento & $\begin{array}{c}\text { Resistencia a } \\
\text { la compresión } \\
(\mathrm{MPa})\end{array}$ & $\begin{array}{c}\text { Peso } \\
\text { volumétrico } \\
\mathrm{kg} / \mathrm{m}^{3}\end{array}$ & $\begin{array}{c}\text { Módulo de } \\
\text { elasticidad } \\
(\mathrm{GPa})\end{array}$ & $\begin{array}{c}\text { Relación de } \\
\text { Poisson }\end{array}$ & $\begin{array}{c}\text { Módulo } \\
\text { de } \\
\text { cortante } \\
(\mathrm{GPa})\end{array}$ & $\begin{array}{c}\text { Esfuerzo de } \\
\text { Fluencia } \\
(\mathrm{MPa})\end{array}$ \\
\hline $\begin{array}{c}\text { Bóvedas } \\
\text { enladrilladas } \\
\text { Arcos de }\end{array}$ & 2 & 1870 & 4 & 0.25 & 1.6 & N/A \\
mampostería & 2 & 1500 & 4 & 0.25 & 1.6 & N/A \\
$\begin{array}{c}\text { Columnas de } \\
\text { mampostería }\end{array}$ & 2 & 1500 & 4 & 0.25 & 1.6 & N/A \\
Vigas de acero & N/A & 7850 & 200 & 0.30 & 77 & 250 \\
\hline
\end{tabular}

\section{Modelo conceptual y condiciones iniciales}

Para lograr los objetivos de esta investigación, dentro del rubro de las simulaciones numéricas se consideró aceptable utilizar en los modelos materiales elástico-lineales con propiedades mecánicas similares a las que utilizó Meli y Sánchez (1993), las cuales son presentadas en la tabla 2. 
Tabla 2. Propiedades mecánicas de los materiales de construcción utilizadas por Meli y Sánchez (1993)

\begin{tabular}{cccccc}
\hline Material & $\begin{array}{c}\text { Peso } \\
\text { volumétrico } \\
(\text { Ton/m3) }\end{array}$ & $\begin{array}{c}\text { Módulo de } \\
\text { elasticidad } \\
(\mathrm{GPa})\end{array}$ & $\begin{array}{c}\text { Relación } \\
\text { de Poisson }\end{array}$ & $\begin{array}{c}\text { Módulo de } \\
\text { cortante } \\
(\mathrm{GPa})\end{array}$ & $\begin{array}{c}\text { Esfuerzo de } \\
\text { fluencia } \\
\text { (MPa) }\end{array}$ \\
\hline $\begin{array}{c}\text { Cantera (riolita y } \\
\text { ignimbrita) de columnas y } \\
\text { arcos }\end{array}$ & 1.5 & 4 & 0.25 & 1.6 & N/A \\
IR de acero & 7.785 & 200 & 0.3 & 77 & 250 \\
\hline
\end{tabular}

De este modo, se desarrollaron una serie de modelos 3-D utilizando los datos de la tendencia de los desplazamientos registrados en los fisurómetros como datos de entrada. Los modelos numéricos se desarrollaron utilizando elementos finitos isoparamétricos, hexaédricos y elástico-lineales de 8 nodos (sólidos) con tres grados de libertad por nodo (desplazamientos lineales en las direcciones $\mathrm{x}, \mathrm{y}, \mathrm{z}$ ). Las dimensiones de las aristas de los elementos variaban entre $300 \mathrm{~mm}$ y $500 \mathrm{~mm}$ aproximadamente, resultando en alrededor de 4,592 elementos, 18,368 nodos para el arco sur y 18,520 para el arco norte. Para el caso de la viga IR, ésta se modeló mediante elementos finitos isoparamétricos rectangulares elástico-lineales de 4 nodos (tipo shell o área). En cada sistema de arco modelado, la base de la cada columna al oeste de la falla se simuló fija al suelo, mientras que a las bases al este de la falla se le permitieron desplazamientos verticales y horizontales, estos últimos solo en el sentido este-oeste. De esta manera el movimiento relativo de la falla se simuló con los desplazamientos de las columnas ubicadas al este de la falla. Es importante mencionar que después de la adaptación estructural del edificio, la incidencia de esfuerzos en los arcos se ha reducido considerablemente, ya que la ahora la función de los arcos es más bien estético-arquitectónica, debido a que, como se mencionó anteriormente, la viga IR colocada es la que ahora resiste todas las demandas estructurales. La figura 7 muestra el ejemplo del modelo elemental de los arcos sur utilizado en las simulaciones numéricas.

a)

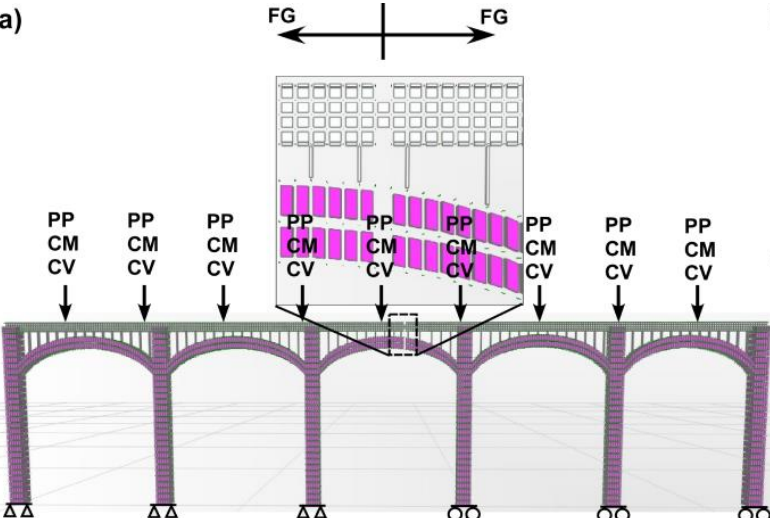

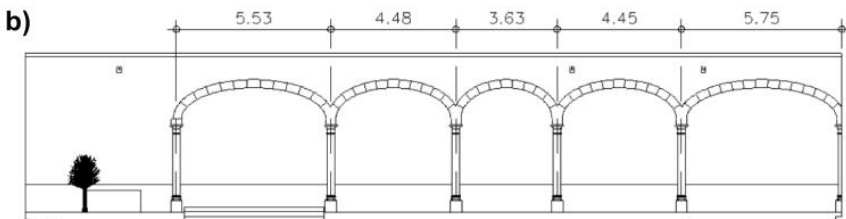

b)

c)

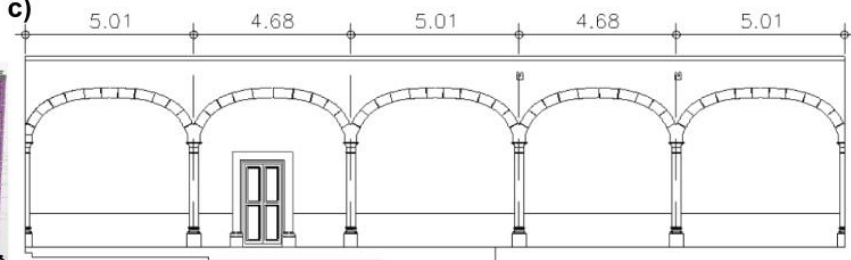

Figura 7. Modelo elemental utilizado en las simulaciones. a) Esquematización de las cargas y apoyos en los arcos sur, en los cuales FG = Acción mecánica inducida en el edificio por los desplazamientos de la falla geológica, $\mathrm{PP}=$ Peso propio de la estructura, $\mathrm{CM}=$ Carga muerta y $\mathrm{CV}=$ Carga viva. $\mathrm{b}$ )

Dimensiones medidas del arco norte en metros. c) Dimensiones en el arco sur en metros. 


\section{RESULTADOS}

\section{Observaciones sobre el arreglo estructural}

En estructuras de mampostería, en general los materiales utilizados en una construcción pueden cambiar con el tiempo. En antiguas construcciones era común que los materiales utilizados se recuperaran de bancos de materiales geológicos cercanos. Este es el caso del Museo de la Insurgencia, ya que está construido básicamente con bloques de roca riolítica unidos mediante juntas de mortero simple a base de cemento-arena, convirtiéndose en una mezcla que resulta en una especie de concreto ciclópeo o concreto sin refuerzo. Sin embargo, como se mencionó anteriormente, no hay información sobre el diseño original y técnicas de construcción, lo que excluye información de la última adaptación estructural que permitió al edificio coexistir hasta la fecha con la falla geológica. Por lo tanto, para obtener información concerniente al arreglo estructural histórico del edificio, particularmente de la condición actual, fue necesario realizar un análisis basado en la observación superficial, ya que, por su condición de edificio patrimonio histórico, tampoco fue posible realizar sondeos exploratorios invasivos. La figura 8 muestra el arco norte, en el cual se resaltan los refuerzos de la última adaptación estructural. Como se puede observar, la viga IR de acero descrita anteriormente se colocó en la parte superior de los arcos para impedir la total separación del edificio, así como permitir que los arcos mantuvieran su curvatura. Estructuralmente dicha viga funciona como un elemento en flexión soportando el peso de los arcos y apoyándose sobre las columnas. Esta viga IR es de aproximadamente unos 20 metros de longitud y se observa a casi todo lo largo de los arcos norte y sur, mismos que son los más afectados por los efectos de la falla superficial.

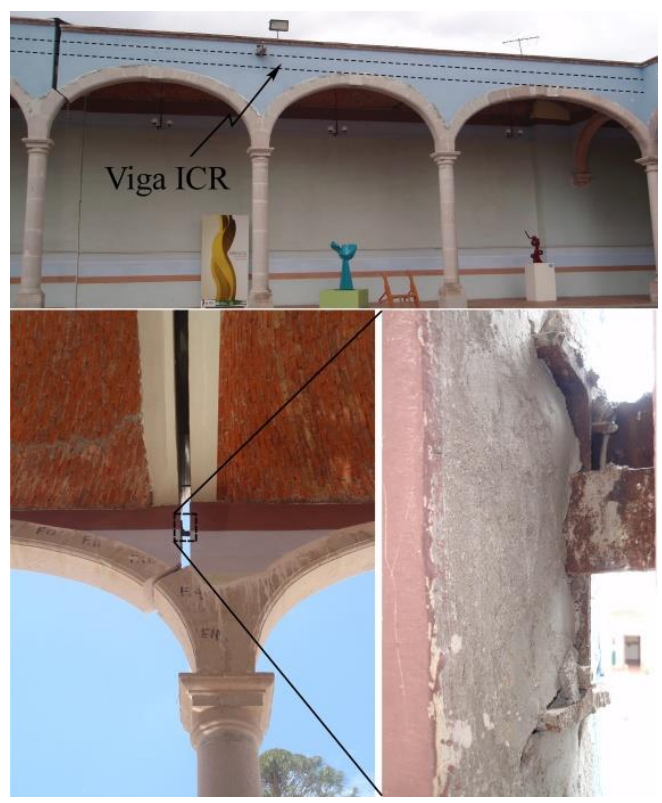

Figura 8. Detalle del arco norte, en el que se puede observar la viga IR colocada sobre los arcos.

\section{Desplazamientos derivados de la falla}

Se tomaron varias lecturas de desplazamiento en los fisurómetros con una frecuencia no mayor a un mes. La figura 9 muestra la dinámica de desplazamiento registrada en los primeros cuatro fisurómetros. Como puede apreciarse, los desplazamientos verticales superan con amplio margen a los horizontales. Además, se puede apreciar una variabilidad espacial y temporal en las magnitudes de los desplazamientos, incluso puede observarse algunas recuperaciones, quizá debido a las características elásticas de los 
materiales constructivos o del suelo, o bien podría tratarse de disminuciones en los esfuerzos propios de la falla. Los máximos desplazamientos verticales y horizontales, están del orden de 10 y 6 milímetros aproximadamente, respectivamente, en acuerdo con lo simulado y medido para otras fallas geológicas en otras investigaciones (Burbey, 2002; Hernández-Marín y Burbey, 2009). Como se mencionó anteriormente, los datos de desplazamiento también fueron usados para calibrar las simulaciones numéricas.

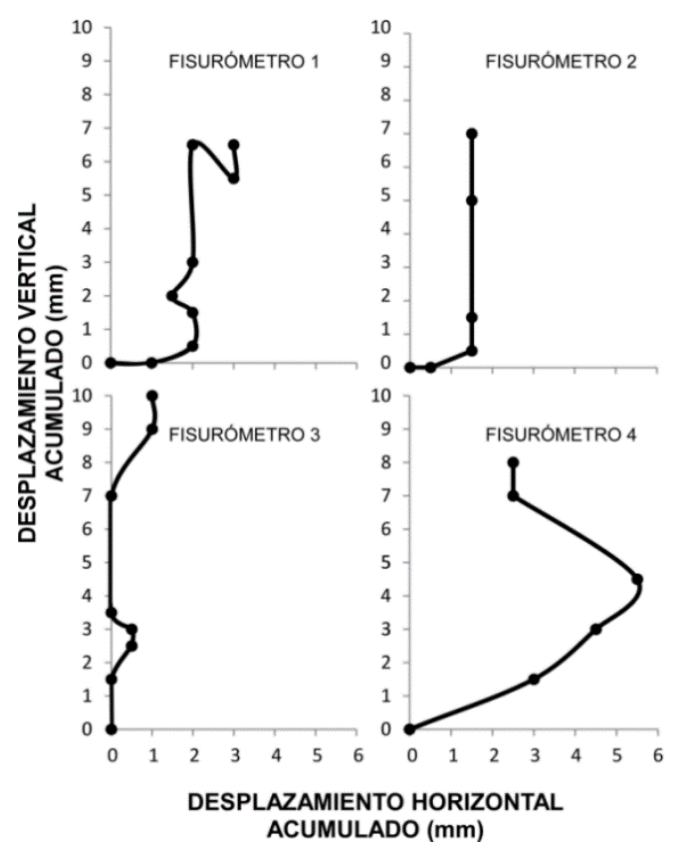

Figura 9. Dinámica de la falla registrada en los primeros cuatro fisurómetros. En esta figura se adecuaron los valores para que iniciara en cero en cada eje coordenado.

\section{Concentración de esfuerzos simulados y su comparativa con las normas actuales}

La figura 10 muestra los resultados de la simulación con respecto a la distribución de esfuerzos normales de tensión-compresión, y referidos al eje ortogonal vertical ante las condiciones de carga estimadas para los arcos norte y sur. Como puede observarse, los esfuerzos mayores se concentran en el extremo inferior de las columnas más cercanas a la junta. Físicamente, la distribución observada de esfuerzos indica que ambas columnas pasan de un estado de compresión en la parte superior a uno de tensión en la base, debido a la presencia de esfuerzos de flexo-tensión.

Las simulaciones permitieron detectar además los esfuerzos resultantes más críticos y se compararon con los permisibles dentro de los estados límite de falla, cuyos valores se obtuvieron de diversas fuentes como lo son las NTCM-RCDF (2004); Meli (1998) y Castellanos (2004). Los resultados se muestran en la tabla 3. 

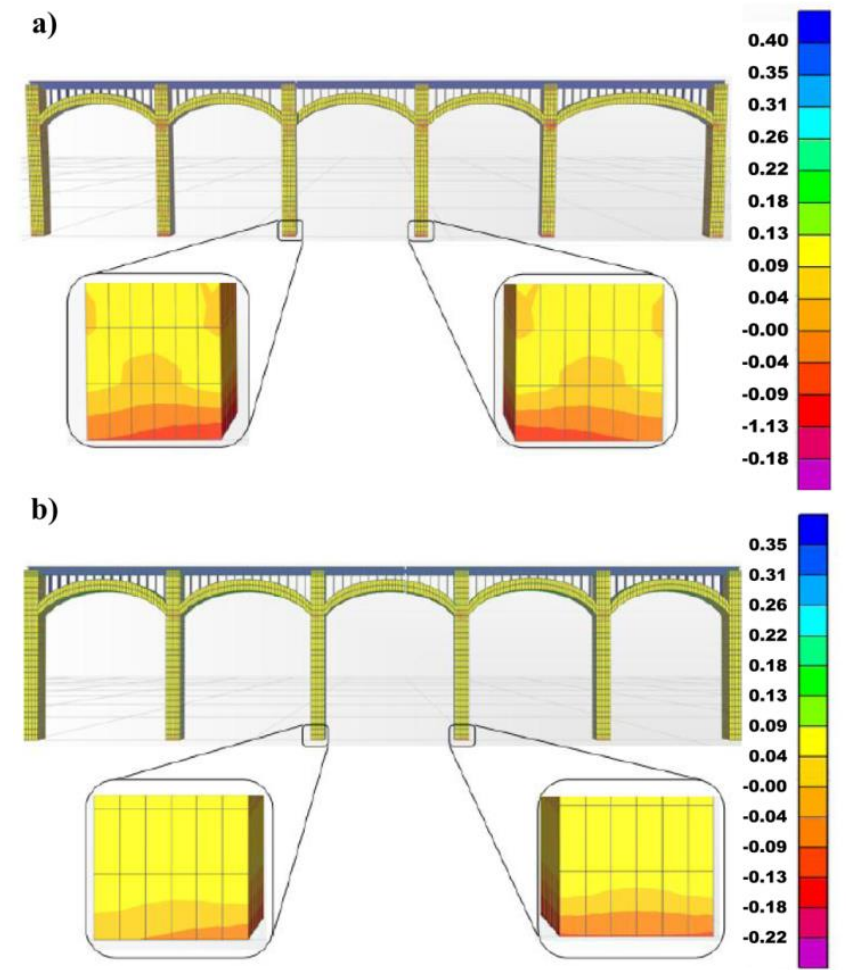

Figura 10. Distribución de esfuerzos normales por la combinación de cargas actuantes. Los valores positivos denotan compresión mientras que los negativos tensión (MPa). a) Arco norte. b) Arco sur.

Tabla 3. Comparaciones entre los esfuerzos críticos simulados y los permitidos dentro del estado límite de falla. Los valores mostrados son magnitudes absolutas

\begin{tabular}{lcccc}
\hline \multirow{2}{*}{ Tipos de esfuerzos } & \multicolumn{3}{c}{ Magnitud del esfuerzo (MPa) } \\
\cline { 3 - 4 } & & \multicolumn{2}{c}{ Valores simulados } & \multirow{2}{*}{$\begin{array}{c}\text { Estado límite de } \\
\text { falla }\end{array}$} \\
\cline { 3 - 4 } Tensión & $\sigma_{\mathrm{x}}$ & 0.12 & $\begin{array}{c}\text { Arcos y } \\
\text { columnas sur }\end{array}$ & \\
\multirow{2}{*}{ Cortante } & $\sigma_{\mathrm{y}}$ & 0.06 & 0.11 & 0.20 \\
Compresión & $\mathrm{T}_{\mathrm{xy}}$ & 0.09 & 0.06 & 0.35 \\
& $\sigma_{\mathrm{x}}$ & 0.25 & 0.31 & 2.00 \\
Esfuerzos & $\sigma_{\mathrm{y}}$ & 0.20 & 0.30 & 0.20 \\
principales & $\sigma_{\min }$ & 0.10 & 0.12 & 2.00 \\
\hline
\end{tabular}

\section{Simulación y análisis de la condición estructural a futuro}

Para complementar el análisis del edificio se realizó una estimación del comportamiento estructural del edificio en un futuro próximo, con base en las deformaciones probables a las que estaría sometida la estructura por la actividad de la falla geológica. Para esto, se utilizaron las tendencias de los desplazamientos relativos registrados en los fisurómetros mediante la estimación lineal de tendencia de los datos registrados durante los 202 días de monitoreo. La tabla 4 resume los datos estimados de la condición hasta un máximo de 50 años. En este caso, la dirección y representa los desplazamientos verticales mientras que la $\mathrm{x}$ representa los desplazamientos horizontales perpendiculares a la traza de la falla. Se vuelve a recalcar que 
la dirección de desplazamientos dominantes es la vertical (eje y), y que en la mayoría de los casos los 680 milímetros serían rebasados en esa dirección vertical a 50 años, siendo el fisurómetro 3 el que alcanzaría un desplazamiento horizontal mayor en ese periodo máximo de estimación. Es de recalcar que de acuerdo con el análisis estadístico de los datos, y a pesar de contar con dispersión considerable en los datos registrados de desplazamiento, el coeficiente de correlación lineal $\mathrm{R}^{2}$ es mayor que 0.5 con excepción de la dirección $\mathrm{x}$ (horizontal perpendicular a la traza de la falla) en el fisurómetro 2, lo que indica buena aproximación de la línea de tendencia, ya que, por su naturaleza impredecible en cuanto a sus desplazamientos y velocidades, el conocer con exactitud la tendencia de una falla geológica como la que afecta a este edificio es prácticamente imposible.

Tabla 4. Estimaciones lineales de desplazamientos relativos en los próximos 50 años a partir de los datos recolectados en los fisurómetros. Se incluye la ecuación que gobierna la línea de tendencia de los desplazamientos en cada dirección en función del tiempo $(\mathrm{t})$

\begin{tabular}{|c|c|c|c|c|c|c|}
\hline $\begin{array}{c}\text { TIEMPO } \\
\text { TRANSCURRIDO }\end{array}$ & $\begin{array}{l}\text { AÑOS } \\
\text { DÍAS }\end{array}$ & $\begin{array}{l}0.5 \\
180\end{array}$ & $\begin{array}{c}1 \\
365\end{array}$ & $\begin{array}{c}5 \\
1825 \\
\end{array}$ & $\begin{array}{c}10 \\
3650\end{array}$ & $\begin{array}{c}50 \\
18250\end{array}$ \\
\hline $\begin{array}{l}\text { FISURÓMETRO } 1 \\
\mathrm{x}=0.0104 \mathrm{t}+3.0547\end{array}$ & Desplazamiento en $\mathrm{x}(\mathrm{mm})$ & 4.9 & 6.9 & 22.0 & 41.0 & 192.9 \\
\hline $\begin{aligned} & \mathrm{R}_{\mathrm{x}}{ }^{2}=0.6569 \\
& \mathrm{y}= 0.0379 \mathrm{t}-1.9331 \\
& \mathrm{R}_{\mathrm{y}}{ }^{2}=0.8384\end{aligned}$ & Desplazamiento en y (mm) & 4.9 & 11.9 & 67.2 & 136.4 & 689.7 \\
\hline $\begin{array}{l}\text { FISURÓMETRO } 2 \\
\mathrm{x}=0.0058 \mathrm{t}+1.142\end{array}$ & Desplazamiento en $\mathrm{x}(\mathrm{mm})$ & 2.2 & 3.3 & 11.7 & 22.3 & 107.0 \\
\hline $\begin{aligned} & \mathrm{R}_{\mathrm{x}}{ }^{2}=0.4955 \\
\mathrm{y}= & 0.0338 \mathrm{t}-2.1764 \\
& \mathrm{R}_{\mathrm{y}}{ }^{2}=0.8266\end{aligned}$ & Desplazamiento en y (mm) & 3.9 & 10.2 & 59.5 & 121.2 & 614.7 \\
\hline $\begin{array}{c}\text { FISURÓMETRO } 3 \\
\mathrm{x}=0.0042 \mathrm{t}+2.1598\end{array}$ & Desplazamiento en $\mathrm{x}(\mathrm{mm})$ & 2.9 & 3.7 & 9.8 & 25.2 & 78.8 \\
\hline $\begin{aligned} & \mathrm{R}_{\mathrm{x}}{ }^{2}=0.7527 \\
\mathrm{y}= & 0.0474 \mathrm{t}-0.4718 \\
& \mathrm{R}_{\mathrm{y}}{ }^{2}=0.9708\end{aligned}$ & Desplazamiento en y (mm) & 8.1 & 16.8 & 86.0 & 259.0 & 864.6 \\
\hline $\begin{array}{l}\text { FISUROMETRO } 4 \\
\mathrm{x}=0.0149 \mathrm{t}+2.2521\end{array}$ & Desplazamiento en $\mathrm{x}(\mathrm{mm})$ & 4.9 & 7.7 & 29.4 & 56.6 & 274.2 \\
\hline $\begin{array}{c}R^{2}(x)=0.9046 \\
y=-0.0469 t+0.4316 \\
R_{y}{ }^{2}=0.9265\end{array}$ & Desplazamiento en y (mm) & 8.9 & 17.6 & 86.0 & 171.6 & 856.4 \\
\hline
\end{tabular}

Una vez determinados los desplazamientos inducidos por la falla a futuro, se procedió a simular los esfuerzos derivados para cada uno de los periodos considerados, que son $0.5,1,5,10$ y 50 años. Se encontró que de acuerdo con las condiciones de desplazamiento de la tabla 4, a partir de los 5 años el edificio sobrepasa los estados límite de falla para varios tipos de esfuerzo (véase tabla 5), siendo los esfuerzos de tensión los más críticos los cuales podrían resultar físicamente en grietas en la estructura de mampostería, como ya se aprecian en la base de una de las columnas de los arcos norte (figura 11). Por otro lado, en la figura 12 se observa, con base en los resultados numéricos, que también se genera una concentración de esfuerzos de tensión en la base de las columnas. Los esfuerzos tensionales simulados en la base al parecer han resultado en fracturamiento como se observa más adelante en la figura 11. En este caso dichas simulaciones no tienen como fin la reproducción de los daños a nivel de fisuras, ya que para esto sería necesario utilizar modelos constitutivos no lineales, sino solamente detectar zonas críticas en función de la magnitud y concentración de los esfuerzos. Es de recalcarse que la presente es una aproximación basada 
solamente en esfuerzos derivados de las tendencias de desplazamiento por efecto de la falla, dichas tendencias corresponden a un ajuste lineal de los datos de desplazamiento medidos.

Tabla 5. Comparaciones entre los esfuerzos críticos simulados y los permitidos dentro del estado límite de falla para un periodo de 5 años a partir de la toma de datos. Los valores mostrados son magnitudes absolutas

\begin{tabular}{|c|c|c|c|c|}
\hline \multirow{2}{*}{\multicolumn{2}{|c|}{ Tipos de esfuerzos }} & \multicolumn{3}{|c|}{ Magnitud del esfuerzo (MPa) } \\
\hline & & \multicolumn{2}{|c|}{ Valores simulados } & \multirow{2}{*}{$\begin{array}{l}\text { Estado límite de } \\
\text { falla }\end{array}$} \\
\hline & & $\begin{array}{c}\text { Arcos y columnas } \\
\text { norte }\end{array}$ & $\begin{array}{c}\text { Arcos y } \\
\text { columnas sur }\end{array}$ & \\
\hline \multirow{2}{*}{ Tensión } & $\sigma_{\mathrm{x}}$ & $0.38 *$ & $0.23 *$ & \multirow{2}{*}{0.00} \\
\hline & $\sigma_{\mathrm{y}}$ & $0.23 *$ & $0.27 *$ & \\
\hline Cortante & $\mathrm{T}_{\mathrm{xy}}$ & $0.36^{*}$ & $0.36 *$ & 0.35 \\
\hline \multirow{2}{*}{ Compresión } & $\sigma_{\mathrm{x}}$ & 0.10 & 0.13 & \multirow{2}{*}{2.00} \\
\hline & $\sigma_{\mathrm{y}}$ & 0.46 & 0.14 & \\
\hline \multirow{2}{*}{$\begin{array}{l}\text { Esfuerzos } \\
\text { principales }\end{array}$} & $\sigma_{\min }$ & 0.15 & $0.23 *$ & 0.20 \\
\hline & $\sigma_{\max }$ & 0.80 & 0.83 & 2.00 \\
\hline
\end{tabular}

*Estos valores sobrepasan los valores del estado límite de falla.

Figura 11. Fisura en la base de una columna en el arco norte. Foto tomada a finales de esta investigación (agosto 2013).

\section{DISCUSIÓN}

Es válido agregar en esta sección que se reconoce el esfuerzo realizado por las autoridades para mantener un edificio en pie por su rol histórico y gran importancia patrimonial en México. El haber intentado separar el edifico en dos bloques para que la falla coexista con el edificio parece ser una buena solución, con adecuaciones menores como la colocación de una junta fría, aunque como se mostró en el patio central del edificio, la traza de una falla puede variar con el tiempo. Sin embargo, al no concretarse una total separación del edificio, por la incrustación de dos vigas en las líneas de arcos norte y sur, los más directamente afectados por la falla, se está impidiendo el libre desplazamiento del edificio por la actividad discontinua de la falla. 


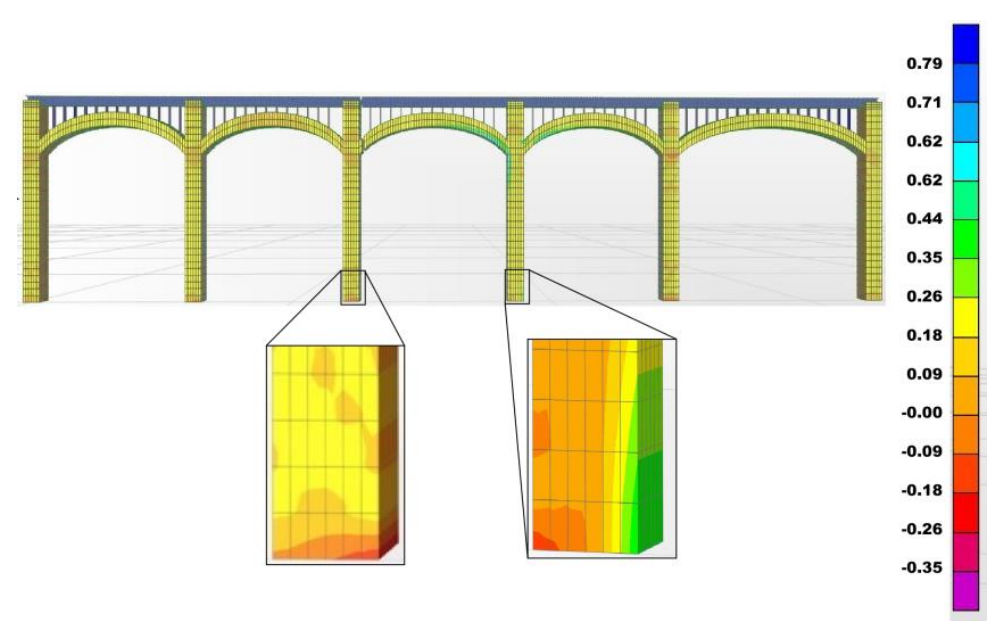

Figura 12. Arcos y columnas norte con desplazamientos relativos a 5 años de acuerdo con los datos registrados. Los valores positivos denotan compresión mientras que los negativos tensión. Las unidades son MPa.

El caso del museo de la Insurgencia presenta una condición en la que una estructura se ha adecuado para coexistir con una falla superficial activa. La estrategia fue simple: dividir el edificio en dos bloques para permitir que la dinámica de la falla no cause daños estructurales graves. Sin embargo, con el fin de preservar el diseño arquitectónico original, se implantaron vigas IR sobre dos líneas de $\operatorname{arcos}$ de mampostería, las cuales mantienen unidos a los dos bloques a través de estos arcos, que son los arcos norte y sur del edificio, permitiendo que los esfuerzos se sigan acumulando y que la falla siga causando daño. Por lo tanto, se plantea la siguiente pregunta: ¿Es necesario mantener de manera estricta el diseño original? Con base en los resultados de este trabajo, puede establecerse que un peligro de colapso sigue latente y continua incrementándose por la resistencia propia de la viga. Las simulaciones del comportamiento del edificio a futuro mostraron que de acuerdo a la tendencia de los desplazamientos relativos registrados, algunas zonas del edificio, en particular las bases de las columnas, se acercan un estado estructural crítico, aunque esta advertencia debe tomarse con reserva, debido a que esta advertencia está basada en los resultados de los análisis, que a su vez se basaron en los registros de desplazamientos relativos producidos por la falla geológica, a sabiendas de la actividad de una falla puede ser muy heterogénea no sólo espacialmente, sino temporalmente, por lo que las tendencias de desplazamiento estimadas en este trabajo pueden variar en el futuro. Sin embargo, independientemente de potenciales cambios en la velocidad de desplazamiento o magnitud de los esfuerzos, el peligro de colapso del edificio podría presentarse a mediano plazo y deberá tomarse en cuenta para futuras adecuaciones.

\section{CONCLUSIONES}

La falla que afecta al edificio analizado tuvo desplazamientos máximos de 10 y 6 milímetros, horizontal y verticalmente, respectivamente, en un lapso de medición de 202 días. Este dato, junto con la resistencia propia de la viga unida por una solera, está provocando la concentración de esfuerzos en las bases de las columnas de esas dos líneas de arcos. Estos esfuerzos ya empiezan a mostrar sus daños por medio de grietas en las bases de estas columnas, las cuales, de acuerdo a simulaciones numéricas de análisis lineal de esfuerzo, en conjunto con las condiciones de desplazamiento medidas, la estructura podría presentar un estado estructural crítico y posible colapso a mediano plazo, siempre y cuando la tendencia de desplazamiento de la falla sea igual o muy similar a la registrada en los fisurómetros colocados en el edificio. Una posible solución a este problema sería cambios en la geometría del diseño de los arcos como por ejemplo agregar una nueva columna que permita la total separación en dos bloques del inmueble. 


\section{AGRADECIMIENTOS}

Los autores agradecen los comentarios, sugerencias y correcciones de los revisores de este artículo, ya que ayudaron a mejorar considerablemente la calidad del mismo. María de Jesús Láriz reconoce y agradece la beca otorgada por el Consejo Nacional de Ciencia y Tecnología (CONACYT) para la culminación de su maestría. Finalmente se agradece la disposición de la directora y empleados del Museo de la Insurgencia, que otorgaron las facilidades para realizar el estudio.

\section{REFERENCIAS}

Aranda-Gómez, J J (1989), "Geología preliminar del graben de Aguascalientes", Revista Mexicana de Ciencias Geológicas, Vol. 8, No. 1, pp. 22-32.

Aranda-Gómez, J M y J J Aranda-Gómez (1985), "Análisis del agrietamiento en la ciudad de Aguascalientes", Reporte de la Universidad Autónoma de Aguascalientes, 108 p.

Burbey, T J, (2002), "The Influence of faults in basins-fill deposits on land subsidence, Las Vegas Valley, Nevada, USA”, Hydrogeology Journal, Vol. 10, No. 5, pp. 525-538. DOI: 10.1007/s10040-002-0215$\underline{7}$

Castellanos, H. (2004), "Efecto del agrietamiento en la respuesta sísmica de un templo colonial típico", Tesis de maestría, Universidad Nacional Autónoma de México, México.

Creazza G, R Matteazzi, A Saetta y R Vitalini, (2002), "Analyses of masonry vaults: a macro approach based on three-dimensional damage model", Journal of Structural Engineering, vol. 128, núm. 5, pp. 646-654. DOI: 10.1061/(ASCE)0733-9445(2002)128:5(646).

Hernández-Marín, M y T J Burbey (2009), "The role of faulting on surface deformation patterns from pumping-induced groundwater flow (Las Vegas Valley, USA)", Hydrogeology Journal, Vol. 17, No. 8, pp. 1859-1875. DOI: $10.1007 / \mathrm{s} 10040-009-0501-8$.

Láriz-Medina M J (2013), “Análisis de daños estructurales en edificaciones por discontinuidades geológicas superficiales en la comunidad de Pabellón de Hidalgo, Rincón de Romos, Aguascalientes”, Tesis de Maestría, Universidad Autónoma de Aguascalientes, Aguascalientes, 219 p.

López J, S Oller y E Oñate (1998), Cálculo del comportamiento de la mampostería mediante elementos finitos, Centro Internacional de Métodos Numéricos en Ingeniería, Monografía No. 46, Barcelona, p. 3.

Lourenço P B (2001), “Analysis of historical constructions: from thrust-lines to advanced simulations”, en Paulo B. Lourenço y Pere Roca (ed.), Historical Constructions, 3er Intern. Symp. Guimarães, 7-9, University of Minho, pp. 91-116

Meli R (1998), Ingeniería estructural de los edificios históricos, Fundación ICA, México D.F., p. 4.

Meli, R y A R Sánchez (1993), "Rehabilitación de la Catedral de la Ciudad de México, México", Reporte del Instituto de Ingeniería para la Dirección General de Sitios y Monumentos del Patrimonio, Nacional, SEDESOL, 87 p.

Meli, R y A R Sánchez (1996), "Considerations on the seismic safety of historical monuments", 11th World Conference on Earthquake Engineering, Paper No. 2087, Elsevier Science Ltd.

NTCM-RCDF (2004), "Normas Técnicas Complementarias para Diseño y Construcción de Estructuras de Mampostería, Reglamento de Construcciones del Distrito Federal", Gaceta Oficial del Distrito Federal, Tomo I, No. 103-BIS, octubre. 

de Aguascalientes

Nuestro México.com. (2013). http://www.nuestro-mexico.com/Aguascalientes/Rincon-deRomos/Pabellon-de-Hidalgo/ .

Ortíz-Lozano, J A, F Alonso, J Pacheco-Martínez, M E Zermeño-de-León, G Araiza y E Mendoza-Otero (2010), "Assessment of the state of condition of damaged buildings and structures affected by land subsidence". Eight International Congress on Land Subsidence (EISOLS), Santiago de Querétaro, México), Octubre 2010, IAHS Publication 339, pp. 449-451.

Orduña, A, G Roeder y F Peña (2007). "Evaluación sísmica de construcciones históricas de mampostería: comparación de tres modelos de análisis", Revista de Ingeniería Sísmica, No. 77, pp. 71-88. DOI: $\underline{10.18867 / 2007-R I S-77-4}$

Pacheco-Martínez, J, M Hernández-Marín, T J Burbey, N González-Cervantes, J A Ortíz-Lozano, M E Zermeño-de-León y A Solís-Pinto, (2013), "Land subsidence and ground failure associated to groundwater exploitation in the Aguascalientes valley, Mexico". Engineering Geology, Vol. 164, No. 0, pp. 172-186. DOI: 10.1016/j.enggeo.2013.06.015.

Peña-Mondragón, F (2010). "Estrategias para el modelado y el análisis sísmico de estructuras históricas", Revista de Ingeniería Sísmica, No. 83, pp. 43-63. DOI: 10.18867/2010-\%25x

Secretaría de Obras Públicas (2010). Portal del Estado de Aguascalientes. En http://www.aguascalientes.gob.mx/sop/sifagg/web/Documentos/Introduccion.pdf . 